\title{
ALMOST WELL-DOMINATED BIPARTITE GRAPHS WITH MINIMUM DEGREE AT LEAST TWO
}

\author{
Hadi Alizadeh AND Didem GÖZÜPEK*
}

\begin{abstract}
A dominating set in a graph $G=(V, E)$ is a set $S$ such that every vertex of $G$ is either in $S$ or adjacent to a vertex in $S$. While the minimum cardinality of a dominating set in $G$ is called the domination number of $G$ denoted by $\gamma(G)$, the maximum cardinality of a minimal dominating set in $G$ is called the upper domination number of $G$ denoted by $\Gamma(G)$. We call the difference between these two parameters the domination gap of $G$ and denote it by $\mu_{d}(G)=\Gamma(G)-\gamma(G)$. While a graph $G$ with $\mu_{d}(G)=0$ is said to be a well-dominated graph, we call a graph $G$ with $\mu_{d}(G)=1$ an almost well-dominated graph. In this work, we first establish an upper bound for the cardinality of bipartite graphs with $\mu_{d}(G)=k$, where $k \geq 1$, and minimum degree at least two. We then provide a complete structural characterization of almost well-dominated bipartite graphs with minimum degree at least two. While the results by Finbow et al. [Ars Comb. 25A (1988) 5-10] imply that a 4-cycle is the only well-dominated bipartite graph with minimum degree at least two, we prove in this paper that there exist precisely 31 almost well-dominated bipartite graphs with minimum degree at least two.
\end{abstract}

Mathematics Subject Classification. 05C69, 05C75, 68R10.

Received July 9, 2019. Accepted April 23, 2020.

\section{INTRODUCTION}

A dominating set in a graph $G=(V, E)$ is a set $S$ such that every vertex of $G$ is either in $S$ or adjacent to a vertex in $S$. A dominating set is called minimal if no proper subset of it is a dominating set. A dominating set of minimum size is called a minimum dominating set. While the cardinality of a minimum dominating set is referred to as domination number of $G$ and is denoted by $\gamma(G)$, the maximum cardinality of a minimal dominating set is called the upper domination number of $G$ and is denoted by $\Gamma(G)$. We call the difference between these two parameters as the domination gap of $G$ and denote it by $\mu_{d}(G)=\Gamma(G)-\gamma(G)$.

Finbow et al. [2] first introduced the concept of well-dominated graphs, which are graphs whose minimal dominating sets have the same cardinality. In other words, a graph $G$ is well-dominated if and only if $\mu_{d}(G)=0$. Well-dominated graphs were further studied in [4]. Finbow et al. [2] provided two characterizations: one for welldominated graphs of girth at least five and the other for well-dominated bipartite graphs. Note that a graph $G$ belongs to the family $\mathcal{P}$ if every vertex of $G$ is either a leaf or is a vertex adjacent to exactly one leaf. Finbow et al. [2] showed that a bipartite well-dominated graph is either in the family $\mathcal{P}$ or is a 4-cycle. Further characterization results in the literature are for well-dominated block graphs and unicyclic graphs [9], locally

Keywords. Bipartite graphs, domination gap, well-dominated graphs, almost well-dominated graphs.

Department of Computer Engineering, Gebze Technical University, Kocaeli, Turkey.

*Corresponding author: didem.gozupek@gtu.edu.r 
well-dominated graphs and locally independent well-dominated graphs [10], 3-connected, planar, and claw-free well-dominated graphs [5], 4-connected, 4-regular, claw-free well-dominated graphs [3], and well-dominated graphs containing neither 4-cycles nor 5-cycles [6].

We say that a graph $G$ is almost well-dominated if and only if $\mu_{d}(G)=1$. With this definition, almost well-dominated (AWD) graphs fall into the class of $D_{2}$ graphs defined by Dunbar et al. [1]. According to Dunbar et al. [1], the class $D_{n}$ consists of graphs having minimal dominating sets with exactly $n$ distinct sizes. In accordance with this notation, $D_{2}$ is the class of graphs having minimal dominating sets with exactly two different sizes. Dunbar et al. [1] gave a characterization for a subclass of bipartite graphs in $D_{2}$ having a vertex adjacent to more than one leaf.

In this paper, we focus on bipartite graphs with domination gap $k$ and minimum degree at least two. We first show that for a fixed value of $k$, there exists a finite number of bipartite graphs with domination gap $k$ and minimum degree at least two by establishing an upper bound for the order of such bipartite graphs. We then provide a complete characterization of almost well-dominated bipartite graphs with minimum degree at least two. Note that in the case of $\mu_{d}(G)=0$ and minimum degree at least two, there exists a single bipartite graph, which is a 4-cycle [2]. In this paper, we extend this result by showing that for $\mu_{d}(G)=1$ and minimum degree at least two, the number of bipartite graphs is precisely 31.

\section{Preliminaries}

A graph $G$ is an ordered pair $(V(G), E(G))$, where $V(G)$ is the set of vertices and $E(G)$ is the set of edges each connecting a pair of vertices. Throughout this paper, $G$ is a simple graph, that is, a finite, undirected, and loopless graph without multiple edges. While the number of vertices of a graph is called the order of the graph, the set of all vertices that are adjacent to a vertex $v$ is called the neighborhood of $v$, and is denoted by $N(v)$. The cardinality of $N(v)$ is called the degree of vertex $v$, which we denote by $\mathbf{d}_{v}$ in this paper. Furthermore, by $\delta(G)$ (resp. $\Delta(G)$ ), we denote the minimum (resp. maximum) degree of $G$.

We frequently use the following lemmas, which show that the domination number of graphs with minimum degree at least 2 and 3 is restricted by an upper bound. Note that in Lemma 2.1, $\mathcal{A}$ is the family of graphs shown in Figure 1.

Lemma 2.1 ([7]). Let $G$ be a connected graph with $\delta(G) \geq 2$ and $G \notin \mathcal{A}$. Then $\gamma(G) \leq\lfloor 2 n / 5\rfloor$.

Among the graphs in the family $\mathcal{A}$, the only bipartite graphs are $A_{5}$ and $A_{6}$. While $A_{5}$ is a well-dominated graph, $A_{6}$ is an almost well-dominated bipartite graph where the minimal dominating sets have size either three or four. The result [8] in Lemma 2.2 establishes an upper bound for the domination number of connected graphs with minimum degree at least three.

Lemma $2.2([8])$. Let $G$ be a connected graph with $\delta(G) \geq 3$. Then $\gamma(G) \leq\lfloor 3 n / 8\rfloor$.

In Lemma 2.3, we state our result on bipartite graphs with $\mu_{d}(G)=k$ and $\delta(G) \geq 2$.

Lemma 2.3. Let $G$ be a bipartite graph with $\delta(G) \geq 2$ and $\mu_{d}(G)=k$, where $k \geq 1$. Then $|V(G)| \leq 10 k$.

Proof. Let $G$ be a bipartite graph with $\delta(G) \geq 2$ and $\mu_{d}(G)=k$ and let $A$ and $B$ be the partite sets of $G$, where $|A| \geq|B|$ and $|V(G)|=n$. By Lemma 2.1, $G$ has a minimal dominating set $D_{1}$ of size at most $\lfloor 2 n / 5\rfloor$. Note that in a connected bipartite graph every partite set is a minimal dominating set. Hence, since $|A|+|B|=n$ and $|A| \geq|B|$, we have that $|A| \geq n / 2$, implying that $G$ has another minimal dominating set $D_{2}$ of size at least $n / 2$. Since the domination gap of $G$ is $k,\left|D_{2}\right|-\left|D_{1}\right| \leq k$. On the other hand, $\left|D_{2}\right|-\left|D_{1}\right| \geq n / 2-\lfloor 2 n / 5\rfloor$. Thus, we have that $n / 2-\lfloor 2 n / 5\rfloor \leq k$, which yields $n \leq 10 k$. It remains to check the exceptional graphs in the family $\mathcal{A}$, where the only bipartite graph with $\mu_{d} \geq 1$ is $A_{6}$. It is easy to see that $\mu_{d}\left(A_{6}\right)=1$ and its order is 7 . Hence, $\left|V\left(A_{6}\right)\right| \leq 10$.

An immediate implication of Lemma 2.3 for almost well-dominated graphs is stated in the following corollary. 


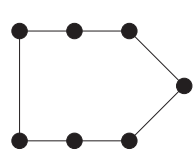

$A_{1}$

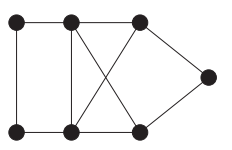

$A_{4}$

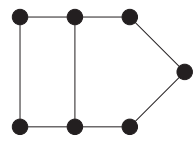

$A_{2}$

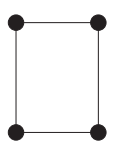

$A_{5}$

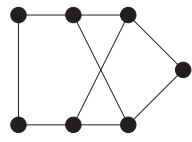

$A_{3}$

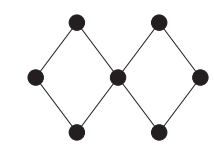

$A_{6}$

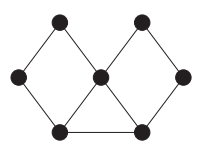

$A_{7}$

FiguRE 1 . The graphs in family $\mathcal{A}$.

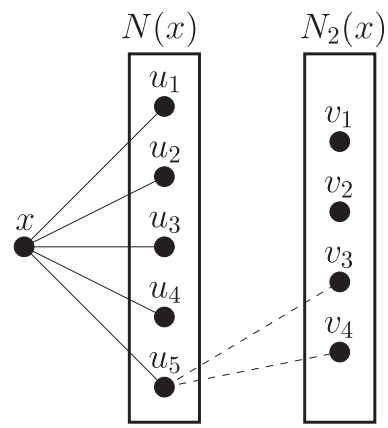

Figure 2. The graph $G$ with a vertex $x$ of degree 5 .

Corollary 2.4. Let $G$ be an almost well-dominated bipartite graph with $\delta(G) \geq 2$. Then $|V(G)| \leq 10$.

While the result in Corollary 2.4 restricts the order of almost well-dominated bipartite graphs with $\delta(G) \geq 2$ by 10 , the following lemma establishes an upper bound for the maximum degree of each vertex in almost well-dominated bipartite graphs with minimum degree 2 .

Lemma 2.5. Let $G$ be an almost well-dominated bipartite graph with $\delta(G) \geq 2$. Then $\Delta(G) \leq 4$.

Proof. Let $G$ be an almost well-dominated bipartite graph with $\delta(G) \geq 2$. Assume to the contrary that there exists a vertex $x$ with degree at least 5 in $G$, i.e., $|N(x)| \geq 5$. Let $N_{i}(x)$ be the set of vertices at distance $i$ from $x$. We first show that $N_{i \geq 3}(x)=\emptyset$. Suppose to the contrary that $N_{3}(x) \neq \emptyset$. Then $N(x) \cup N_{3}(x)$ is an independent set of size at least 6 , which implies a maximal independent (minimal dominating) set $D_{1}$ of size at least 6 . However, by Corollary 2.4, $G$ has at most 10 vertices and thus, by Lemma $2.1, \gamma(G) \leq 4$. This contradicts with $G$ being almost well-dominated since $\left|D_{1}\right|-\gamma(G) \geq 2$ and thus $N_{i \geq 3}(x)=\emptyset$.

Note that $x \cup N_{2}(x)$ and $N(x)$ are two minimal dominating sets in $G$. If $|N(x)| \geq 6$, then $N(x)$ is a minimal dominating set of size at least 6 , contradiction with $G$ being almost well-dominated. Therefore, $N(x)$ is of size exactly 5 (see Fig. 2). Since $G$ is an almost well-dominated graph and $|V(G)| \leq 10$, the possible size for $x \cup N_{2}(x)$ is either 5 or 4 , i.e., the possible sizes of $N_{2}(x)$ are 3 and 4 . If $\left|N_{2}(x)\right|=3$, then $|V(G)|=9$, which implies that $\gamma(G) \leq 3$ and $|N(x)|-\gamma(G) \geq 2$, contradiction.

Now consider the case where $\left|N_{2}(x)\right|=4$. Suppose for a contradiction that a vertex $u_{1}$ in $N(x)$ has three neighbors $\left\{v_{1}, v_{2}, v_{3}\right\}$ in $N_{2}(x)$. Then the set $\left\{x, u_{1}, v_{4}\right\}$ forms a dominating set of size three; however, the domination gap becomes 2 since $N(x)$ is a minimal dominating set of size 5 , contradiction. Therefore, in this case any vertex in $N(x)$ has at most two neighbors in $N_{2}(x)$. Furthermore, since each vertex in $N_{2}(x)$ has degree 
at least two, there are at least 8 edges between $N(x)$ and $N_{2}(x)$. Since $|N(x)|=5$, by pigeonhole principle, there exists a vertex in $N(x)$, say $u_{5}$, with two neighbors in $N_{2}(x)$. Without loss of generality, let $\left\{v_{3}, v_{4}\right\}$ be the two neighbors of $u_{5}$ in $N_{2}(x)$. Each of the vertices $v_{1}$ and $v_{2}$ have at least two neighbors in $N(x)$. The vertex $u_{5}$ is not among the possible neighbors for these vertices since it has its own two neighbors $\left\{v_{3}, v_{4}\right\}$ in $N_{2}(x)$. Here, two cases arise: neighborhoods of $v_{1}$ and $v_{2}$ are either disjoint or not. In the first case, let $\left\{u_{1}, u_{2}\right\}$ and $\left\{u_{3}, u_{4}\right\}$ be the disjoint neighborhoods of $v_{1}$ and $v_{2}$ in $N(x)$, respectively. In this case, the set $\left\{u_{5}, v_{1}, v_{2}\right\}$ forms a dominating set of size 3 , contradiction. In the latter case, let $u_{2}$ be a common neighbor for $v_{1}$ and $v_{2}$ in $N(x)$. Then the set $\left\{x, u_{5}, u_{2}\right\}$ forms a dominating set of size 3 , contradiction.

The following result determines the upper domination number of an almost well-dominated bipartite graph.

Lemma 2.6. Let $G$ be a connected almost well-dominated bipartite graph. Then $G$ has two partite sets $A$ and $B$ with $|A| \geq|B|$ such that $|A|-|B| \leq 1$ and $\Gamma(G)=|A|$.

Proof. Let $G$ be a connected bipartite graph with $A$ and $B$ as its partite sets. Observe that each partite is a minimal dominating set. Hence $|A|-|B| \leq 1$. It remains to prove that $\Gamma(G)=|A|$. Suppose to the contrary that there exists a minimal dominating set $D$ of size at least $|A|+1$. Let $D^{\prime}=V(G)-D$. Every vertex in $D$ has at least one neighbor in $D^{\prime}$ since $D$ is a minimal dominating set; therefore, $D^{\prime}$ is a dominating set including a minimal dominating set $D^{\prime \prime}$ of size at most $\left|D^{\prime \prime}\right| \leq\left|D^{\prime}\right|=n-|D| \leq n-|A|-1 \leq|A|-1$. Thus, $|D|-\left|D^{\prime \prime}\right| \geq 2$, a contradiction.

\section{AWD Bipartite Graphs With $\delta(G) \geq 2$}

By the aid of the Corollary 2.4 and Lemma 2.5, we give a complete structural characterization of almost well-dominated bipartite graphs with $\delta(G) \geq 2$. We first focus on the case of bipartite almost well-dominated graphs with $\delta(G) \geq 3$ and then proceed with the case $\delta(G)=2$.

\subsection{AWD bipartite graphs with $\delta(G) \geq 3$}

In the case of $\delta(G) \geq 3$, Lemma 2.1 provides an upper bound for the domination number of graphs with $\delta(G) \geq 3$. Using this upper bound, we state the result on almost well-dominated bipartite graphs with $\delta(G) \geq 3$ in Lemma 3.1.

Lemma 3.1. Let $G$ be a connected bipartite almost well-dominated graph with $\delta(G) \geq 3$. Then $G$ is isomorphic to $G_{6-1}$ in Figure 4.

Proof. Notice that, by Lemma 2.6, an almost well-dominated bipartite graph $G$ of even order $n$ has two partite sets, each of size $n / 2$, and that of odd order $n$ has two partite sets with size $(n+1) / 2$ and $(n-1) / 2$. Furthermore, by Lemma $2.2, G$ has a minimal dominating set of size at most $\lfloor 3 n / 8\rfloor$. In the case of odd $n,(n+1) / 2-\lfloor 3 n / 8\rfloor \leq 1$ yields $n \leq 3$, implying that there is no almost well-dominated bipartite graph with $\delta(G) \geq 3$ and odd order. On the other hand, for even $n, n / 2-\lfloor 3 n / 8\rfloor \leq 1$ yields $n \leq 8$. Hence, we focus on the cases with $|V(G)|=6$ and $|V(G)|=8$.

Let $|V(G)|=6$. Then $G$ has two partites each of size three. Let $A=\{a, b, c\}$ and $B=\{d, e, f\}$ be the two partites of $G$. Since $\delta(G) \geq 3$, each vertex has at least three neighbors in the other partite. Since the size of each partite is three, each vertex is adjacent to exactly three vertices in the other partite. The graph obtained in this case is shown as $G_{6-1}$ in Figure 4.

Next assume that $|V(G)|=8$. Then $G$ has two partites each of size four. Let $A=\{a, b, c, d\}$ and $B=\{e, f, g, h\}$ be the two partites of $G$. Both $A$ and $B$ are minimal dominating sets of size 4 . We show that $G$ has a minimal dominating set of size two and hence $G$ is not almost well-dominated. Note that, by Lemma 2.5, the vertices in $G$ have degrees either 3 or 4 . Suppose that a vertex of $G$, say $a$, is of degree 4 . Then the total degree of the vertices in partite $A$ is at least 13, implying that there exists at least one vertex of degree 4 in the partite $B$, say $e$. Then $\{a, e\}$ forms a minimal dominating set of size two, contradiction. On the other 
hand, if $a$ is of degree three, it has three neighbors, say $\{e, f, g\}$, in partite $B$ and since $h$ has degree at least 3 , it is adjacent to $\{b, c, d\}$, implying that $\{a, h\}$ is a minimal dominating set of size two, contradiction.

\subsection{AWD bipartite graphs with $\delta(G)=2$}

Recall that an almost well-dominated bipartite graph $G$ of even order $n$ has two partite sets, each of size $n / 2$, and the one with odd order $n$ has two partite sets with sizes $(n+1) / 2$ and $(n-1) / 2$. Furthermore, by Lemma 2.1, $G$ has a minimal dominating set of size at most $\lfloor 2 n / 5\rfloor$. Note that in the case of odd $n$, we may assume that $G$ has two minimal dominating sets $D_{1}$ of size $(n+1) / 2$ and $D_{2}$ of size at most $\lfloor 2 n / 5\rfloor$, respectively. Since we are interested in graphs with $\mu_{d}=1$, we have that $\left|D_{1}\right|-\left|D_{2}\right| \leq 1$. In other words, $(n+1) / 2-\lfloor 2 n / 5\rfloor \leq 1$, implying that $n \leq 5$. Notice further that while for odd $n \geq 7,\left|D_{1}\right|-\left|D_{2}\right| \geq 2$, a bipartite graph with $\delta=2$ has at least 4 vertices. Thus, for the case of almost well-dominated bipartite graphs with $\delta=2$ and odd order, we have to investigate graphs with order 5 together with the only exceptional bipartite graph with odd order in the family $\mathcal{A}$, namely $A_{6}$ of order 7 (see Fig. 1) for which Lemma 2.1 does not hold. On the other hand, for even $n$, we may assume that $G$ has two minimal dominating sets $D_{1}$ of size $n / 2$ and $D_{2}$ of size at most $\lfloor 2 n / 5\rfloor$, respectively. Since $\mu_{d}=1$, it follows that $\left|D_{1}\right|-\left|D_{2}\right| \leq 1$, which in turn implies that $n \leq 10$. For $|V(G)|=4$, there is a single bipartite graph with $\delta(G)=2$, that is $C_{4}$, which is not almost well-dominated. Therefore, for the case of almost well-dominated bipartite graphs with $\delta=2$ and even order, we must check orders 6,8 , and 10. Hence, from now onwards, we will focus on orders $5,6,7,8$, and 10 separately and prove that there are precisely 30 almost well-dominated bipartite graphs with $\delta(G)=2$ (see Fig. 4).

The case $|V(G)|=5$ leads to a single almost well-dominated graph. This result is stated in Lemma 3.2.

Lemma 3.2. Let $G$ be a connected bipartite almost well-dominated graph with $\delta(G)=2$ and $|V(G)|=5$. Then $G$ is isomorphic to $G_{5}$ in Figure 4 .

Proof. Let $G$ be a connected bipartite almost well-dominated graph with $\delta(G)=2$ and $|V(G)|=5$. Then $G$ has two partites $A$ and $B$ of sizes 3 and 2, respectively. Since $\delta(G)=2$, every vertex in $A$ is adjacent to both vertices in $B$. This case results in the graph $G_{5}$ shown in Figure 4.

We next assume that $|V(G)|=6$. This case yields 3 almost well-dominated bipartite graphs. Lemma 3.3 states the result of this case.

Lemma 3.3. Let $G$ be a connected almost well-dominated bipartite graph with $\delta(G)=2$ and $|V(G)|=6$. Then $G \in\left\{G_{6-2}, G_{6-3}, G_{6-4}\right\}$ in Figure 4 .

Proof. Here $G$ has two partites each of size three. Let $A=\{a, b, c\}$ and $B=\{d, e, f\}$ be the two partites of $G$. In this case, a vertex can be adjacent to at most three vertices in the other partite; hence, $\Delta(G) \leq 3$. Suppose first that $\Delta(G)=2$. Then all vertices of $G$ are of degree 2 . Hence $G$ is isomorphic to $C_{6}$, which is shown as $G_{6-2}$ in Figure 4. Now suppose that $\Delta(G)=3$. Note that there is at least one vertex of degree two in $G$. Let $a$ in the partite $A$ be such a vertex. Then partite $A$ has at least a vertex of degree 3 since otherwise all vertices of $A$ would be of degree 2 implying that all vertices in $B$ are of degree two. Let $\mathbf{d}_{c}=3$. Then $b$ has degree either 2 or 3 . We consider these two possible degrees for $b$ separately in the sequel.

Let $\mathbf{d}_{b}=2$, i.e., $\left(\mathbf{d}_{a}, \mathbf{d}_{b}, \mathbf{d}_{c}\right)=(2,2,3)$. This degree sequence for partite $A$ implies the same degree sequence for partite $B$, i.e., $\left(\mathbf{d}_{d}, \mathbf{d}_{e}, \mathbf{d}_{f}\right)=(2,2,3)$. Hence, vertices $c$ and $f$ are adjacent to all vertices in $B$ and $A$, respectively. The vertex $a$ is adjacent to one of $\{d, e\}$, say $d$. Hence $b$ is adjacent to $e$. The graph obtained here is an almost well-dominated graph shown as $G_{6-3}$ in Figure 4.

Next suppose that $\mathbf{d}_{b}=3$, i.e., $\left(\mathbf{d}_{a}, \mathbf{d}_{b}, \mathbf{d}_{c}\right)=(2,3,3)$. This degree sequence for partite $A$ implies that $\left(\mathbf{d}_{d}, \mathbf{d}_{e}, \mathbf{d}_{f}\right)=(2,3,3)$ for partite $B$. In this case, the vertices $b$ and $c$ are adjacent to all vertices in partite $B$ and the vertices $e$ and $f$ are adjacent to all vertices in partite $A$. The graph obtained here is an almost well-dominated graph shown as $G_{6-4}$ in Figure 4 . 
In the case $|V(G)|=7$, we obtain a single almost well-dominated bipartite graph. Lemma 3.4 states our result for $|V(G)|=7$.

Lemma 3.4. Let $G$ be a connected almost well-dominated bipartite graph with $\delta(G)=2$ and $|V(G)|=7$. Then $G$ is isomorphic to $G_{7}$, shown in Figure 4 .

Proof. Let $G$ be a bipartite graph of order 7 such that $G \notin \mathcal{A}$ (shown in Fig. 1). Then by Lemma $2.1, \gamma(G) \leq 2$ for $n=7$. Furthermore, $G$ has two partites of sizes 4 and 3 . Hence $\mu_{d}(G) \geq 2$, a contradiction. Next we assume that $G \in \mathcal{A}$. Among the graphs in the family $\mathcal{A}$, the only bipartite graph on 7 vertices is $A_{6}$, which is an almost well-dominated graph shown as $G_{7}$ in Figure 4.

Lemmas 3.7 and 3.8 state the results for the case $|V(G)|=8$. Propositions 3.5 and 3.6 provide us tools to characterize almost well-dominated bipartite graphs with $\delta(G)=2$ and order 8 .

Proposition 3.5. Let $G$ be a connected almost well-dominated bipartite graph with $\delta(G)=2$ and $|V(G)|=8$. Then any two vertices of degree 3 in different partites of $G$ are adjacent.

Proof. Note that $G$ has two partites each of size 4 . Let $A=\{a, b, c, d\}$ and $B=\{e, f, g, h\}$ be the two partites of $G$. Suppose to the contrary that $a$ and $e$ are two nonadjacent vertices of degree three in different partites. Then $a$ is adjacent to the vertices in $\{f, g, h\}$ and $e$ is adjacent to the vertices in $\{b, c, d\}$. Then $\{a, e\}$ is a minimal dominating set of size two. However, each partite set of $G$ is a minimal dominating set of size 4 and hence $\mu_{d}(G) \geq 2$, contradiction to $G$ being almost well-dominated.

Proposition 3.6. Let $G$ be a connected almost well-dominated bipartite graph with $\delta(G)=2$ and $|V(G)|=8$. Then

(i) There exists no two vertices of degree 4 in different partites.

(ii) There exist at most two vertices of degree 4 in one partite.

Proof. Note that $G$ has two partites each of size 4 . For (i), it is easy to see that two vertices of degree 4 in different partites form a minimal dominating set of size two, a contradiction with $G$ being almost well-dominated. For (ii), suppose to the contrary that there exist at least three vertices of degree 4 in one partite, say partite $A$. Then the sum of the degrees of the vertices in partite $A$ is at least 14 which enforces the existence of at least two vertices of degree 4 in the partite $B$, a contradiction to (i).

From here onwards, we focus on the cases with $\Delta(G) \leq 3$ and $\Delta(G)=4$, separately. For $\Delta(G) \leq 3$, Lemma 3.7 and for $\Delta(G)=4$, Lemma 3.8 state the main results for almost well-dominated bipartite graphs with $\delta(G)=2$ and $|V(G)|=8$.

Lemma 3.7. Let $G$ be a connected almost well-dominated bipartite graph with $\delta(G)=2, \Delta(G) \leq 3$, and $|V(G)|=8$. Then $G \in\left\{G_{8-5}, G_{8-6}, G_{8-7}, G_{8-8}, G_{8-9}\right\}$ shown in Figure 4.

Proof. Note that each vertex of $G$ has degree 2 or 3 . If all vertices have degree two, $G \cong C_{8}$, which is an almost well-dominated graph shown as $G_{8-5}$ in Figure 4 . Hence, we proceed with $\Delta(G)=3$. Note that $G$ has two partites each of size 4 . Let $A=\{a, b, c, d\}$ and $B=\{e, f, g, h\}$ be the two partites of $G$. Observe that if all vertices in one partite have degree 3 (or 2 ) then all vertices in the other partite have degree 3 (or 2) as well, a contradiction with $\delta(G)=2$ and $\Delta(G)=3$. Hence, each partite $A$ and $B$ has at least one vertex with degree 3 and one with degree 2 . Let $\mathbf{d}_{a}=3$ and $\mathbf{d}_{b}=2$ in $A$. Then the degrees of the remaining two vertices, namely $\left(\mathbf{d}_{c}, \mathbf{d}_{d}\right)$ is either $(2,2),(2,3)$, or $(3,3)$. We proceed with the proof by considering the possible sequences for $\left(\mathbf{d}_{c}, \mathbf{d}_{d}\right)$ separately in the sequel.

Assume first that $\left(\mathbf{d}_{c}, \mathbf{d}_{d}\right)=(2,2)$, i.e., $\left(\mathbf{d}_{a}, \mathbf{d}_{b}, \mathbf{d}_{c}, \mathbf{d}_{d}\right)=(3,2,2,2)$. This degree sequence for partite $A$ implies that $\left(\mathbf{d}_{e}, \mathbf{d}_{f}, \mathbf{d}_{g}, \mathbf{d}_{h}\right)=(3,2,2,2)$. By Proposition 3.5, the vertices of degree 3 in different partites, namely $a$ and $e$ are adjacent. Furthermore, $a$ has two other neighbors in $\{f, g, h\}$, say $\{f, g\}$. Similarly, $e$ is 
adjacent to two other vertices in $\{b, c, d\}$, say $\{b, c\}$. We first assume that $d h \in E(G)$. Then $d$ is adjacent to one of $\{f, g\}$, say $g$. Similarly, $h$ is adjacent to one of $\{b, c\}$, say $c$. Then $f$ is adjacent to $b$. The graph obtained in this case is shown as $G_{8-6}$ in Figure 4. Next suppose that $d h \notin E(G)$. Then $d$ is adjacent to both $f$ and $g$, and $h$ is adjacent to both $b$ and $c$. The graph obtained here is shown as $G_{8-9}$ in Figure 4.

Assume next that $\left(\mathbf{d}_{c}, \mathbf{d}_{d}\right)=(3,2)$, i.e., $\left(\mathbf{d}_{a}, \mathbf{d}_{b}, \mathbf{d}_{c}, \mathbf{d}_{d}\right)=(3,2,3,2)$. This degree sequence for partite $A$ implies that $\left(\mathbf{d}_{e}, \mathbf{d}_{f}, \mathbf{d}_{g}, \mathbf{d}_{h}\right)=(3,2,3,2)$. By Proposition 3.5, vertices of degree three in partite $A$, namely $a$ and $c$ are adjacent to the vertices of degree 3 in partite $B$, namely $e$ and $g$. We proceed with the proof based on the possible connections between vertices of degree two in the sequel.

Suppose first that there exists at least one vertex of degree two with two neighbors of degree two. Let $b$ in partite $A$ be such a vertex adjacent to the vertices in $\{f, h\}$. Then the vertices $e$ and $g$ are adjacent to $d$ as their third neighbor. Further, $a$ is adjacent to one of $\{f, h\}$, say $f$. Hence, $c$ is adjacent to $h$. This case results in $G_{8-8}$ depicted in Figure 4.

Suppose next that a vertex of degree two is adjacent to at most one vertex of degree two. In this case, we first show that there exists at least one pair of adjacent vertices of degree two. Suppose to the contrary that there exists no two adjacent vertices of degree two. Then each vertex of degree 2 has two neighbors of degree 3 . Then the resulting graph is disconnected, contradiction. Thus, there is at least one pair of adjacent vertices of degree two. Let $b$ and $f$ be such a pair. Since $b$ is not adjacent to another vertex of degree two, it is adjacent to one of the vertices of degree three $\{e, g\}$, say $e$. Similarly $d$ is not adjacent to $f$ and hence it is adjacent to both $g$ and $h$. Further, $f$ is adjacent to one of $\{a, c\}$, say $a$, and finally $c$ is adjacent to $h$. This case yields $G_{8-7}$ depicted in Figure 4.

For the last case, we assume that $\left(\mathbf{d}_{c}, \mathbf{d}_{d}\right)=(3,3)$, i.e., $\left(\mathbf{d}_{a}, \mathbf{d}_{b}, \mathbf{d}_{c}, \mathbf{d}_{d}\right)=(3,2,3,3)$. This degree sequence for partite $A$ implies that $\left(\mathbf{d}_{e}, \mathbf{d}_{f}, \mathbf{d}_{g}, \mathbf{d}_{h}\right)=(3,2,3,3)$. By Proposition 3.5, each of the vertices $a, c$, and $d$ are adjacent to each of the vertices $e, g$, and $h$. Hence, $b$ and $f$ remain disconnected. Thus, this case does not lead to any connected almost well-dominated bipartite graph.

Lemma 3.8. Let $G$ be a connected almost well-dominated bipartite graph with $\delta(G)=2, \Delta(G)=4$, and $|V(G)|=8$. Then $G \in\left\{G_{8-1}, G_{8-2}, G_{8-3}, G_{8-4}\right\}$ shown in Figure 4.

Proof. Let $G$ be an almost well-dominated bipartite graph with $\delta(G)=2, \Delta(G)=4$, and $|V(G)|=8$. Let further $A=\{a, b, c, d\}$ and $B=\{e, f, g, h\}$ be the two partites of $G$. By Proposition 3.6, there are at most two vertices of degree 4 in one partite and no two vertices of degree 4 in different partites.

First suppose that $G$ has two vertices of degree 4, say $a$ and $b$. Then, by Proposition 3.6, the vertices in partite $B$ are of degree either 2 or 3 . Note that the sum of the degrees of the vertices in partite $A$ is at least 12 , while the corresponding sum for partite $B$ is at most 12 . Thus, the only possible degrees for $c$ and $d$ is 2 , i.e., $\left(\mathbf{d}_{a}, \mathbf{d}_{b}, \mathbf{d}_{c}, \mathbf{d}_{d}\right)=(4,4,2,2)$. This degree sequence for partite $A$ implies that $\left(\mathbf{d}_{e}, \mathbf{d}_{f}, \mathbf{d}_{g}, \mathbf{d}_{h}\right)=(3,3,3,3)$ for partite $B$. Note that the vertices of degree 4 , namely $a$ and $b$, are both adjacent to all vertices in partite $B$. The vertex $c$ is adjacent to any two vertices in partite $B$, say $\{e, f\}$. Finally, $d$ is adjacent to $g$ and $h$. The graph obtained here is shown as $G_{8-3}$ in Figure 4.

Next suppose that $G$ has a single vertex of degree 4 , say $a$ in the partite $A$. Note that the vertices in partite $B$ are of degree either 2 or 3 by Proposition 3.6. Since the sum of the degrees of the vertices in partite $B$ is at most 12 , the possible degree sequences for partite $A$ are $(4,2,2,2),(4,3,2,2)$, and $(4,3,3,2)$. We address these cases separately in the sequel.

Suppose first that $\left(\mathbf{d}_{a}, \mathbf{d}_{b}, \mathbf{d}_{c}, \mathbf{d}_{d}\right)=(4,2,2,2)$. With this degree sequence for partite $A$, the only possible degree sequence for the partite $B$ is $(2,2,3,3)$. Without loss of generality suppose that $\left(\mathbf{d}_{e}, \mathbf{d}_{f}, \mathbf{d}_{g}, \mathbf{d}_{h}\right)=$ $(2,2,3,3)$. Naturally, $a$ is adjacent to all vertices in partite $B$. Then $h$ is adjacent to any two vertices from $\{b, c, d\}$, say $\{c, d\}$. Now, $b$ is adjacent to at least one of $\{e, f\}$, say $e$. Now consider the second neighbor of $b$ in partite $B$. Suppose first that $b f \in E(G)$. Then $g$ is adjacent to $c$ and $d$. Hence, $G \cong G_{8-4}$ shown in Figure 4 . Now assume that $b g \in E(G)$. Then $g$ is adjacent to one of $c$ or $d$, say $d$. Then $f c \in E(G)$ and $G \cong G_{8-1}$ shown in Figure 4. 
In the case of $\left(\mathbf{d}_{a}, \mathbf{d}_{b}, \mathbf{d}_{c}, \mathbf{d}_{d}\right)=(4,3,2,2)$, partite $B$ has the degree sequence $\left(\mathbf{d}_{e}, \mathbf{d}_{f}, \mathbf{d}_{g}, \mathbf{d}_{h}\right)=(2,3,3,3)$. Naturally, $a$ is adjacent to all vertices in partite $B$. Then $b$ is adjacent to all vertices of degree 3 in $B$, namely $\{f, g, h\}$, by Proposition 3.5. Then $e$ is adjacent to one of $\{c, d\}$, say $c$. Then $c$ is adjacent to one vertex in $\{f, g, h\}$, say $f$. Then $d$ is adjacent to $g$ and $h$. This case yields the almost well-dominated graph $G_{8-2}$ in Figure 4.

Assume next that $\left(\mathbf{d}_{a}, \mathbf{d}_{b}, \mathbf{d}_{c}, \mathbf{d}_{d}\right)=(4,3,3,2)$. In this case, partite $B$ has the degree sequence $\left(\mathbf{d}_{e}, \mathbf{d}_{f}, \mathbf{d}_{g}, \mathbf{d}_{h}\right)=(3,3,3,3)$. By Proposition 3.5 , a vertex of degree 3 in one partite $A$, say $b$, has to be adjacent to all vertices of degree 3 in partite $B$; however, there exist 4 vertices of degree 3 in $B$, contradiction. This case does not lead to any almost well-dominated graph.

Let us now focus on the case where $|V(G)|=10$. In this case, $G$ has two partites each of size 5 . By Lemma 2.5, $\Delta(G) \leq 4$. We focus on the cases with $\Delta(G) \leq 3$ and $\Delta(G)=4$ separately in Lemmas 3.9 and 3.10, respectively.

Lemma 3.9. Let $G$ be a connected almost well-dominated bipartite graph with $\delta(G)=2, \Delta(G) \leq 3$, and $|V(G)|=10$. Then $G \in\left\{G_{10-1}, G_{10-2}, G_{10-3}, G_{10-7}, G_{10-10}, G_{10-12}, G_{10-13}, G_{10-14}, G_{10-15}, G_{10-16}\right\}$.

Proof. Let $A=\{a, b, c, d, e\}$ and $B=\{f, g, h, i, j\}$ be the two partites of $G$. Notice that each partite $A$ or $B$ is a minimal dominating set of size 5 . Thus, a minimal dominating set of size at most 3 contradicts with $G$ being almost well-dominated. Moreover, the degrees of the vertices of $G$ are either 2 or 3 . If all vertices have degree two, then $G$ is isomorphic to a $C_{10}$, which is an almost well-dominated bipartite graph shown as $G_{10-1}$ in Figure 4 . Thus, we assume that there exists at least one vertex with degree three in $G$. Note that if all vertices in one partite have degree 2 (or 3 ), then the vertices in the other partite all have degree 2 (or 3 ). Since $\delta(G)=2$, we may suppose that there are two vertices of degree 3 and 2 in one partite, say $a$ and $b$, respectively, in partite $A$. Now, the degrees of the remaining three vertices, namely $\left(\mathbf{d}_{c}, \mathbf{d}_{d}, \mathbf{d}_{e}\right)$, are either $(2,2,2),(2,2,3),(2,3,3)$, or $(3,3,3)$. In what follows, we consider these cases separately.

Claim 1. Let $G$ be a connected almost well-dominated bipartite graph with $\delta(G)=2, \Delta(G)=3$, and $|V(G)|=10$. If $\left(\mathbf{d}_{a}, \mathbf{d}_{b}, \mathbf{d}_{c}, \mathbf{d}_{d}, \mathbf{d}_{e}\right)=(3,2,2,2,2)$, then $G \in\left\{G_{10-2}, G_{10-14}\right\}$.

Proof of Claim 1. Let $\left(\mathbf{d}_{a}, \mathbf{d}_{b}, \mathbf{d}_{c}, \mathbf{d}_{d}, \mathbf{d}_{e}\right)=(3,2,2,2,2)$. This degree sequence for partite $A$ implies the same degree sequence for partite $B$. Without loss of generality, assume that $\left(\mathbf{d}_{f}, \mathbf{d}_{g}, \mathbf{d}_{h}, \mathbf{d}_{i}, \mathbf{d}_{j}\right)=(3,2,2,2,2)$.

First assume that af $\notin E(G)$. Then $a$ has three neighbors of degree 2 in the partite $B$, say $\{g, h, i\}$. Similarly, $f$ has three neighbors in $\{b, c, d, e\}$, say $\{b, c, d\}$. Note that $e$ is not adjacent to $j$ since otherwise $\{a, f, e\}$ forms a minimal dominating set of size 3 , a contradiction. Thus, $e$ has two neighbors from $\{g, h, i\}$, say $\{h, i\}$. Furthermore, $j$ has two neighbors from $\{b, c, d\}$, say $\{c, d\}$. Finally, $b$ is adjacent to $g$. This case results in $G_{10-14}$ in Figure 4.

Now suppose that $a f \in E(G)$. Then $a$ has two other neighbors from $\{g, h, i, j\}$, say $\{g, h\}$. Similarly, $f$ has two other neighbors from $\{b, c, d, e\}$, say $\{b, c\}$. Since $f$ is adjacent to all vertices $\{a, b, c\}$, we show that $d$ and $e$ do not dominate all vertices $\{g, h, i, j\}$. Suppose to the contrary that $\{d, e\}$ dominates $\{g, h, i, j\}$. Then $\{f, d, e\}$ forms a minimal dominating set of size 3 , contradiction. Thus, $d$ and $e$ have at least one common neighbor from $\{g, h, i, j\}$ which is either $i$ or $j$. Let $i$ be the common neighbor of $d$ and $e$. Similarly, since $a$ is adjacent to the vertices $\{f, g, h\}$, the vertices $i$ and $j$ must not dominate $\{b, c, d, e\}$ in order to avoid a minimal dominating set $\{a, i, j\}$ of size 3 . This implies that $i$ and $j$ have at least one common neighbor in $\{b, c, d, e\}$ which can be either $d$ or $e$. Let $e$ be the common neighbor of $i$ and $j$. Note that $j$ is not adjacent to $d$ since otherwise $G$ is disconnected. Therefore, $j$ is adjacent to one of $\{b, c\}$, say $c$. Then $d$ is adjacent to one of $\{g, h\}$, say $h$. Finally, $b$ is adjacent to $g$. This case yields $G_{10-2}$ depicted in Figure 4 .

Claim 2. Let $G$ be a connected almost well-dominated bipartite graph with $\delta(G)=2, \Delta(G)=3$, and $|V(G)|=10$. If $\left(\mathbf{d}_{a}, \mathbf{d}_{b}, \mathbf{d}_{c}, \mathbf{d}_{d}, \mathbf{d}_{e}\right)=(3,2,2,2,3)$ then $G \in\left\{G_{10-3}, G_{10-7}, G_{10-12}, G_{10-13}\right\}$. 


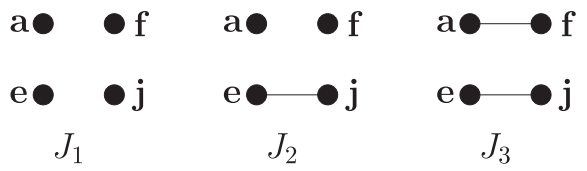

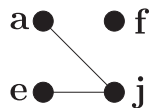

$J_{4}$

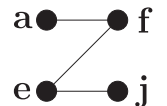

$J_{5}$

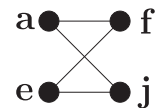

$J_{6}$

Figure 3. The graph $H_{3}$ induced by the vertices of degree three.
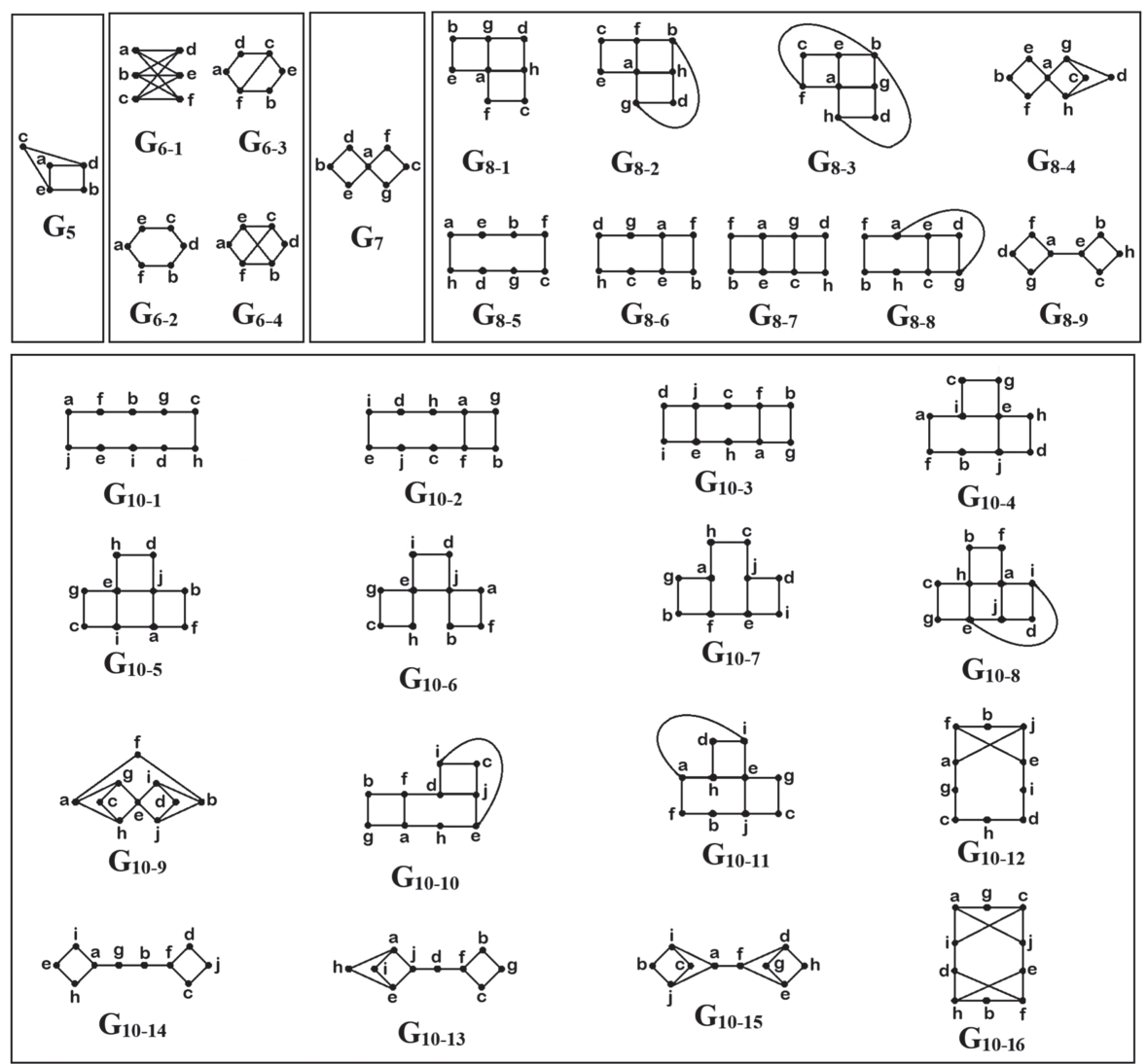

FiguRE 4. AWD bipartite graphs with $\delta(G) \geq 2$.

Proof of Claim 2. Let $\left(\mathbf{d}_{a}, \mathbf{d}_{b}, \mathbf{d}_{c}, \mathbf{d}_{d}, \mathbf{d}_{e}\right)=(3,2,2,2,3)$. This degree sequence for partite $A$ implies that $\left(\mathbf{d}_{f}, \mathbf{d}_{g}, \mathbf{d}_{h}, \mathbf{d}_{i}, \mathbf{d}_{j}\right)=(3,2,2,2,3)$. Let further $H_{3}$ be the subgraph induced by the vertices of degree 3 , namely $\{a, e, f, j\}$. Figure 3 shows the six possible cases for $H_{3}$. We proceed the proof by analyzing each of these six cases separately in the sequel.

If $H_{3} \cong J_{1}$, the vertices of degree 3 are adjacent to all three vertices of degree 2 in the other partite. The resulting graph is disconnected, contradiction. Next assume that $H_{3} \cong J_{2}$. In this case, each of $a$ and $f$ is 
adjacent to all three vertices of degree 2 in the other partite. Then, $\{a, f, e\}$ is minimal dominating set of size 3 , contradiction.

We then suppose that $H_{3} \cong J_{3}$. In this case, $a$ has two neighbors from $\{g, h, i\}$, say $\{g, h\}$. Similarly, $f$ has two neighbors from $\{b, c, d\}$, say $\{b, c\}$. Now we claim that $i$ and $j$ do not dominate $\{b, c, d, e\}$. Suppose to the contrary that $i$ and $j$ dominate $\{b, c, d, e\}$. Then $\{a, i, j\}$ forms a minimal dominating set of size three, contradiction. Note that the neighbors of $i$ and $j$ are from the set $\{b, c, d, e\}$ which has size 4 . Since $j$ has degree 3 , the vertex $i$ has two common neighbors with $j$, since otherwise $i$ and $j$ dominate $\{b, c, d, e\}$. The common neighbors of $i$ and $j$ are $d$ and $e$. Now, as its third neighbor, $e$ is adjacent to one of $\{g, h\}$, say $h$. Similarly, $j$ is adjacent to one of $\{c, b\}$, say $c$. Finally, $g$ is adjacent to $b$ and the graph obtained in this case is $G_{10-3}$ shown in Figure 4.

In the case of $H_{3} \cong J_{4}$, the vertex $f$ is adjacent to $\{b, c, d\}$. We will now show that $a$ and $e$ do not dominate $\{g, h, i, j\}$. Suppose to the contrary that $a$ and $e$ dominate $\{g, h, i, j\}$. Then $\{f, a, e\}$ forms a minimal dominating set of size 3 , a contradiction. Observe that the candidate neighbors of $a$ and $e$ are from the set $\{g, h, i, j\}$. Since $a$ and $e$ are both of degree 3, they must have three common neighbors, since otherwise they dominate the set $\{g, h, i, j\}$. Moreover, note that one of these common neighbors is $j$ and the other two must be selected from $\{g, h, i\}$, say $\{h, i\}$. Then $g$ has two neighbors from $\{b, c, d\}$, say $\{b, c\}$. Finally, $d$ is adjacent to $j$. The graph obtained in this case is $G_{10-13}$ depicted in Figure 4.

Next suppose that $H_{3} \cong J_{5}$. First, $a$ is adjacent to two vertices from $\{g, h, i\}$, say $\{g, h\}$. Similarly, $j$ is adjacent to two vertices from $\{b, c, d\}$, say $\{c, d\}$. The vertex $b$ is not adjacent to $i$ since otherwise $\{a, j, b\}$ forms a minimal dominating set of size 3 , contradiction. Then $b$ has at least one neighbor from $\{g, h\}$, say $g$. Similarly, $i$ has at least one neighbor from $\{c, d\}$, say $d$. At this point, as its third neighbor, $f$ has two candidates, namely $\{b, c\}$. If $f c \in E(G)$, then $b$ is adjacent to $h$ and $\{f, b, d\}$ is a minimal dominating set of size 3, contradiction. Hence, $f$ is adjacent to $b$ as its third neighbor. In addition, $e$ is not adjacent to $h$ since otherwise $i$ is adjacent to $c$ and $\{e, i, g\}$ is a minimal dominating set of size 3, contradiction. Thus, $e$ is adjacent to $i$ as its third neighbor. Finally, $c$ is adjacent to $h$. This case leads to almost well-dominated graph $G_{10-7}$ in Figure 4.

Finally, in the case of $H_{3} \cong J_{6}$, the vertex $f$ is adjacent to one of $\{b, c, d\}$, say $b$, as its third neighbor. Similarly, $e$ is adjacent to one of $\{g, h, i\}$, say $i$, as its third neighbor. We now show that the vertices $c$ and $d$ do not dominate $\{g, h, i, j\}$. Suppose to the contrary that $c$ and $d$ do not dominate $\{g, h, i, j\}$. Then $\{f, c, d\}$ is a minimal dominating set of size 3 , contradiction. Thus, $c$ and $d$ do not dominate $\{g, h, i, j\}$. As $c$ and $d$ are both of degree two, they have at least one common neighbor in order not to dominate $\{g, h, i, j\}$, where the only candidates are $g$ and $h$. Let $h$ be the common neighbor of $c$ and $d$. At this point, notice that $g$ is not adjacent to both $a$ and $b$, since otherwise $\{g, h, e\}$ forms a minimal dominating set of size 3 , contradiction. Therefore, $g$ has at least one neighbor from $\{c, d\}$. Let $c$ be the neighbor of $g$. The vertices $d$ and $g$ are not adjacent since otherwise the graph is disconnected. Observe that if $b g \in E(G)$ and $i d \in E(G)$, then $j$ and $a$ remain without a third neighbor, contradiction. Hence, at least one of $b g \notin E(G)$ and $i d \notin E(G)$ must hold. By symmetry, suppose that $b g \notin E(G)$. Recall that $g$ is not adjacent to $d$. Therefore, $g$ is adjacent to $a$. Now we show that $i$ is not adjacent to $b$, because otherwise $\{a, h, i\}$ is a minimal dominating set of size 3 , contradiction. Thus, $i$ is adjacent to $d$. Finally, $j$ is adjacent to $b$. The graph obtained in this case is $G_{10-12}$ in Figure 4 .

Claim 3. Let $G$ be a connected almost well-dominated bipartite graph with $\delta(G)=2, \Delta(G)=3$, and $|V(G)|=10$. If $\left(\mathbf{d}_{a}, \mathbf{d}_{b}, \mathbf{d}_{c}, \mathbf{d}_{d}, \mathbf{d}_{e}\right)=(3,2,2,3,3)$ then $G \in\left\{G_{10-10}, G_{10-15}\right\}$.

Proof of Claim 3. Let $\left(\mathbf{d}_{a}, \mathbf{d}_{b}, \mathbf{d}_{c}, \mathbf{d}_{d}, \mathbf{d}_{e}\right)=(3,2,2,3,3)$. This degree sequence for partite $A$ implies that $\left(\mathbf{d}_{f}, \mathbf{d}_{g}, \mathbf{d}_{h}, \mathbf{d}_{i}, \mathbf{d}_{j}\right)=(3,2,2,3,3)$. In this case, we first claim that a vertex of degree two is adjacent to at most one vertex of degree two in the other partite. Suppose to the contrary that $b$ is adjacent to two vertices of degree two in partite $B$, namely $g$ and $h$. We now show that $c$ is adjacent to at least one of $g$ or $h$. Suppose to the contrary that $c$ is adjacent to two vertices of degree 3 , say $\{i, j\}$. Then $f$ is adjacent to all vertices $\{a, d, e\}$ and $\{f, b, c\}$ is a minimal dominating set of size 3, contradiction. Therefore, $c$ is adjacent to at least one of $g$ or $h$, say $g$. At this point, we show that any two vertices of degree 3 in different partites are adjacent. Suppose to the contrary that two vertices of degree 3 in different partites, say $a$ and $f$, are nonadjacent. Then $a$ is adjacent 
to each of $\{h, i, j\}$ and $f$ is adjacent to each of $\{c, d, e\}$ and hence $\{a, f, b\}$ is a minimal dominating set of size 3 , contradiction. Therefore, each vertex of degree 3 in partite $A$, namely $\{a, d, e\}$, is adjacent to each vertex of degree 3 in partite $B$, namely $\{f, i, j\}$. Then, $c$ is adjacent to $h$ and the graph is disconnected, contradiction. Therefore, a vertex of degree two is adjacent to at most one vertex of degree two in the other partite. We proceed with the proof of this case based on the connection between vertices of degree two in the sequel.

Suppose first that no two vertices of degree two are adjacent. In this case $c$ has two neighbors from vertices of degree $3\{f, i, j\}$, say $\{i, j\}$. Similarly, $h$ has two neighbors from $\{a, d, e\}$, say $\{d, e\}$. In addition, $g$ has at least one neighbor from $\{d, e\}$, say $d$. Similarly, $b$ has at least one neighbor from $\{i, j\}$, say $i$. The vertex $d$ is adjacent to one of $\{i, j, f\}$ as its third neighbor. If $d i \in E(G)$, then $f$ is adjacent to the vertices $\{a, b, e\}$ and hence $\{d, f, j\}$ is a minimal dominating set of size 3 , contradiction. Thus, $d i \notin E(G)$. If $d j \in E(G)$, then $f$ is adjacent to the vertices $\{a, b, e\}$ and hence $\{c, d, f\}$ is a minimal dominating set of size 3 , contradiction. Thus, $d j \notin E(G)$ as well. Therefore, $d$ is adjacent to $f$. If the vertices $i$ and $j$ dominate $\{a, b, c, e\}$, then $\{d, i, j\}$ is a minimal dominating set of size 3 , contradiction. Thus, $i$ and $j$ must have 3 common neighbors (same neighborhood) in order not to dominate $\{a, b, c, e\}$. Since $i$ is adjacent to $b$, then $j$ is also adjacent to $b$. On the other hand, vertex $a$ has 3 neighbors in $\{f, g, i, j\}$, where at least one of these neighbors is either $i$ or $j$. However, since $i$ and $j$ have the same neighborhood, both $i$ and $j$ are adjacent to $a$. Furthermore, $e$ is adjacent to both $f$ and $g$ and hence $a$ is adjacent to $f$. This case results in $G_{10-15}$ shown in Figure 4.

Next assume that at least a pair of vertices of degree two, say $b$ and $g$, are adjacent. Then $b$ and $g$ have their second neighbor from vertices of degree 3, say $b f \in E(G)$ and $g a \in E(G)$. Note that if two vertices of degree 3 , one from $\{d, e\}$ and the other from $\{i, j\}$, say $d$ and $i$, are not adjacent, then $d$ is adjacent to the vertices $\{f, h, j\}$ and $i$ is adjacent to the vertices $\{a, c, e\}$. Then $\{d, i, b\}$ is a minimal dominating set of size 3 , contradiction. Thus, both vertices $d$ and $e$ are adjacent to both vertices $i$ and $j$. At this point, suppose that the vertices $\{d, e\}$ are both adjacent to $h$ and the vertices $\{i, j\}$ are both adjacent to $c$. Then the graph is disconnected, contradiction. Therefore, we may suppose that one of the conditions does not hold; in other words, at least one of $d$ or $e$ is not adjacent to $h$, say $d h \notin E(G)$. Hence $d$ is adjacent to $f$. Notice further that since $d$ dominates $\{f, i, j\}$, the vertices $g$ and $h$ must not dominate $\{a, b, c, e\}$ since otherwise $\{d, g, h\}$ is a minimal dominating set of size 3, contradiction. Therefore, $g$ and $h$ have at least one common neighbor, where the only candidate is $a$ and hence $h a \in E(G)$. The vertices $\{a, c\}$ are the candidates for the third neighbors of $i$ and $j$. Since $a$ can be adjacent to only one of $i$ and $j$, then at least one of $i$ and $j$ is adjacent to $c$, say $j c \in E(G)$. Now, if $a i \in E(G)$, then $\{a, j, b\}$ is a minimal dominating set of size 3 , contradiction. Hence $a i \notin E(G)$ and $a$ is adjacent to $f$ as its third neighbor. Finally, $i$ is adjacent to $c$ and further $h$ is adjacent to $e$. The graph obtained in this case is $G_{10-10}$ shown in Figure 4 .

Claim 4. Let $G$ be a connected almost well-dominated bipartite graph with $\delta(G)=2, \Delta(G)=3$, and $|V(G)|=10$. If $\left(\mathbf{d}_{a}, \mathbf{d}_{b}, \mathbf{d}_{c}, \mathbf{d}_{d}, \mathbf{d}_{e}\right)=(3,2,3,3,3)$, then $G$ is isomorphic to $G_{10-16}$.

Proof of Claim 4. Let $\left(\mathbf{d}_{a}, \mathbf{d}_{b}, \mathbf{d}_{c}, \mathbf{d}_{d}, \mathbf{d}_{e}\right)=(3,2,3,3,3)$. This degree sequence for partite $A$ implies that $\left(\mathbf{d}_{f}, \mathbf{d}_{g}, \mathbf{d}_{h}, \mathbf{d}_{i}, \mathbf{d}_{j}\right)=(3,2,3,3,3)$. In this case, we first show that the vertices of degree two, namely $b$ and $g$, are not adjacent. Suppose to the contrary, that $b g \in E(G)$. The vertices $b$ and $g$ have a neighbor from vertices of degree three. Let $b$ be adjacent to $f$ and $g$ be adjacent to $a$. Notice that if two vertices of degree 3 , one from $\{c, d, e\}$ and the other from $\{h, i, j\}$, say $c$ and $h$, are not adjacent, then $c$ is adjacent to $\{f, i, j\}$ and $h$ is adjacent to $\{a, d, e\}$ and hence $\{c, h, b\}$ is a minimal dominating set of size 3 , contradiction. Then each vertex in $\{c, d, e\}$ is adjacent to each vertex in $\{h, i, j\}$ and the graph is disconnected, contradiction. Thus, the vertices $b$ and $g$ are nonadjacent. Therefore, we may suppose that $b$ is adjacent to two vertices of degree 3 , say $\{f, h\}$, and $g$ is adjacent to two vertices in $\{a, c, d, e\}$, say $\{a, c\}$. The vertex $e$ has at least one neighbor in $\{i, j\}$, say $j$ and at least one neighbor in $\{h, f\}$, say $h$. In addition, $j$ has at least one neighbor in $\{c, a\}$, say $c$. We now show that $c$ is not adjacent to $h$. Suppose for a contradiction that $c$ is adjacent to $h$. Then $d$ is adjacent to all vertices $\{f, i, j\}$ and $\{d, h, a\}$ is a minimal dominating set of size 3 , contradiction. Thus, ch $\notin E(G)$. Suppose further that $c f \in E(G)$ and $h a \in E(G)$. Then $d$ is adjacent to $i$ and $\{c, h, d\}$ is a minimal dominating set of size 3 , contradiction. Thus, at least one of $c f \in E(G)$ and $h a \in E(G)$ does not hold. By symmetry, we may assume 
that $h a \notin E(G)$ and hence $h d \in E(G)$. Now if $a$ is adjacent to both $f$ and $i$, then $\{h, a, c\}$ is a minimal dominating set of size 3, contradiction. Hence, $a$ has at least one neighbor different from $f$ and $i$, namely aj $\in E(G)$. Furthermore, $d$ is adjacent to both $f$ and $i$. At this point, we show that $a$ is not adjacent to $f$. Suppose to the contrary that $a$ is adjacent to $f$. Then $c$ is adjacent to $i$ and then $\{c, h, a\}$ is a minimal dominating set of size 3 , contradiction. Thus af $\notin E(G)$ and in turn $a$ is adjacent to $i$ as its third neighbor. If $f$ is adjacent to $c$, then $\{a, h, f\}$ is a minimal dominating set of size 3, contradiction. Hence $f c \notin E(G)$ and $f$ is adjacent to $e$. Finally, $c$ is adjacent to $i$. This case results in $G_{10-16}$ shown in Figure 4.

In summary, if $\Delta(G)=2$, then $G$ is isomorphic to $G_{10-1}$ and in the case of $\Delta(G)=3$, the proof of Lemma 3.9 follows from Claims 1-4.

Lemma 3.10. Let $G$ be a connected almost well-dominated bipartite graph with $\delta(G)=2, \Delta(G)=4$, and $|V(G)|=10$. Then $G \in\left\{G_{10-4}, G_{10-5}, G_{10-6}, G_{10-8}, G_{10-9}, G_{10-11}\right\}$.

Proof. Let $A=\{a, b, c, d, e\}$ and $B=\{f, g, h, i, j\}$ be the two partites of $G$. Since $\Delta(G)=4$, there exists at least one vertex of degree 4 , say $e$, in $G$. Let further the vertices $\{g, h, i, j\}$ be the neighbors of $e$ in partite $B$. Note that if $\mathbf{d}_{f}=4$, then $f$ is adjacent to all vertices in $\{a, b, c, d\}$ and $\{e, f\}$ is a minimal dominating set of size 2, contradiction. Furthermore, if $\mathbf{d}_{f}=3$, then $f$ is adjacent to three vertices in $\{a, b, c, d\}$, say $\{b, c, d\}$. Then, $\{e, f, a\}$ is a minimal dominating set of size 3 , contradiction. Thus, $\mathbf{d}_{f}=2$. Let $a$ and $b$ be the neighbors of $f$ in the partite $A$. Note that since $e$ dominates the vertices $\{g, h, i, j\}$ and $f$ dominates the vertices $\{a, b\}$, if $c$ and $d$ have a common neighbor, say $h$, then $\{e, f, h\}$ is a minimal dominating set of size 3 , contradiction. Thus, $c$ and $d$ have disjoint neighborhoods in $\{g, h, i, j\}$, which implies that $c$ and $d$ are both of degree 2 . Thus far, it is known that $\left(\mathbf{d}_{c}, \mathbf{d}_{d}, \mathbf{d}_{e}, \mathbf{d}_{f}\right)=(2,2,4,2)$, the vertex $e$ is adjacent to the vertices in $\{g, h, i, j\}$, and $f$ is adjacent to the vertices in $\{a, b\}$. We proceed with the proof by focusing on the possible degrees for the remaining vertices $a$ and $b$ in partite $A$ in the sequel.

Suppose first that there exist more than one vertex of degree 4 in one partite. Then at least one of $a$ or $b$, say $a$, is of degree 4. Since $c$ and $d$ have disjoint neighborhoods, we suppose that $c$ is adjacent to two vertices in $\{g, h, i, j\}$, say $g$ and $h$. Then $d$ is adjacent to $i$ and $j$. Note that $a$ is adjacent to $f$ and further has 3 neighbors in $\{g, h, i, j\}$, say $\{h, i, j\}$. Now observe that $a$ dominates $\{f, h, i, j\}$ and $g$ has two neighbors $c$ and $e$ in $A$. If $g$ is further adjacent to $b$, then $\{a, g, d\}$ is a minimal dominating set of size 3, contradiction. Therefore, $g$ is not adjacent to $b$. Hence, $\mathbf{d}_{g}=2$. If the vertex $b$ has a neighbor in $\{i, j\}$, say $i$, then $\{a, g, i\}$ is a minimal dominating set of size 3 , contradiction. Therefore, $b$ has no neighbor in $\{i, j\}$ and hence is adjacent to $h$. Therefore, $\mathbf{d}_{b}=2$ and $\mathbf{d}_{h}=4$. The graph obtained in this case, where $\left(\mathbf{d}_{a}, \mathbf{d}_{b}, \mathbf{d}_{c}, \mathbf{d}_{d}, \mathbf{d}_{e}\right)=(4,2,2,2,4)$ and $\left(\mathbf{d}_{f}, \mathbf{d}_{g}, \mathbf{d}_{h}, \mathbf{d}_{i}, \mathbf{d}_{j}\right)=(2,2,4,3,3)$, is shown as $G_{10-8}$ in Figure 4.

Next suppose that there is only one vertex of degree 4 in one partite, i.e., $\mathbf{d}_{a} \neq 4$ and $\mathbf{d}_{b} \neq 4$. In this case, the possible options for $\left(\mathbf{d}_{a}, \mathbf{d}_{b}\right)$ are $(2,2),(3,2)$, and $(3,3)$. We proceed with analyzing each possible degree sequence separately in the following.

Suppose first that $\left(\mathbf{d}_{a}, \mathbf{d}_{b}\right)=(2,2)$, i.e., $\left(\mathbf{d}_{a}, \mathbf{d}_{b}, \mathbf{d}_{c}, \mathbf{d}_{d}, \mathbf{d}_{e}\right)=(2,2,2,2,4)$, which implies two possible degree sequences $(2,2,2,2,4)$ and $(2,2,2,3,3)$ for the partite $B$. Let first $\left(\mathbf{d}_{f}, \mathbf{d}_{g}, \mathbf{d}_{h}, \mathbf{d}_{i}, \mathbf{d}_{j}\right)=(2,2,2,2,4)$. Notice that at least one of $c$ or $d$, say $d$ is adjacent to $j$. Then $d$ is adjacent to one vertex in $\{g, h, i\}$, say $i$. In addition, $c$ is adjacent to $g$ and $h$ since it has a disjoint neighborhood from $d$. Finally, $j$ is adjacent to $a$ and $b$ as its third and fourth neighbors. The graph obtained here is $G_{10-6}$ shown in Figure 4.

In the case of $\left(\mathbf{d}_{f}, \mathbf{d}_{g}, \mathbf{d}_{h}, \mathbf{d}_{i}, \mathbf{d}_{j}\right)=(2,2,2,3,3)$, since $c$ and $d$ have no common neighbor, $j$ has exactly one neighbor from $\{c, d\}$, say $d$. Then, the third neighbor of $j$ is from $\{a, b\}$, say $b$. Similarly, $i$ has exactly one neighbor from $\{c, d\}$ and its third neighbor is different from $c$ and $d$, i.e., it is adjacent to $a$. Now, if $i$ is adjacent to $d$, then $c$ is adjacent to the vertices in $\{g, h\}$ and $\{i, b, c\}$ is a minimal dominating set of size 3 , contradiction. Therefore, $i d \notin E(G)$ and $i$ is adjacent to $c$ as its third neighbor. Then $c$ is adjacent to one of $g$ or $h$, say $g$. Then $d$ is adjacent to $h$. The graph obtained here is $G_{10-4}$ depicted in Figure 4.

Next let $\left(\mathbf{d}_{a}, \mathbf{d}_{b}\right)=(3,2)$, in other words, $\left(\mathbf{d}_{a}, \mathbf{d}_{b}, \mathbf{d}_{c}, \mathbf{d}_{d}, \mathbf{d}_{e}\right)=(3,2,2,2,4)$. With this degree sequence for partite $A$, there are two possible degree sequences $(2,2,2,3,4)$ and $(2,2,3,3,3)$ for the partite $B$. 
First suppose that $\left(\mathbf{d}_{f}, \mathbf{d}_{g}, \mathbf{d}_{h}, \mathbf{d}_{i}, \mathbf{d}_{j}\right)=(2,2,2,3,4)$. Since $c$ and $d$ have no common neighbor, $j$ is adjacent to only one of $c$ or $d$, say $d$. Furthermore, $j$ is adjacent to $a$ and $b$ as its third and fourth neighbors. Observe that if $i c \notin E(G)$, then $i$ is adjacent to the vertices $\{d, a\}$ and $c$ is adjacent to the vertices $\{g, h\}$. Then, $\{i, c, b\}$ is a minimal dominating set of size 3 , contradiction. Therefore, $i c \in E(G)$ and further $i$ is adjacent to $a$ as its third neighbor. Now $c$ is adjacent to one of $g$ or $h$, say $g$, as its second neighbor and finally, $d$ is adjacent to $h$. The graph obtained here is $G_{10-5}$ shown in Figure 4.

We then suppose that $\left(\mathbf{d}_{f}, \mathbf{d}_{g}, \mathbf{d}_{h}, \mathbf{d}_{i}, \mathbf{d}_{j}\right)=(2,2,3,3,3)$. One of $c$ or $d$, say $c$ is adjacent to $g$. The vertex $a$ is adjacent to two vertices in $\{h, i, j\}$, say $h$ and $i$. Notice that since $j$ is adjacent to only one of $c$ or $d$, the other neighbor of $j$ is $b$. Note further that if $j$ is adjacent to $d$, then $\{a, c, j\}$ is a minimal dominating set of size 3 , contradiction. Therefore, $j d \notin E(G)$ and hence $j$ is adjacent to $c$. Finally, $d$ is adjacent to both $h$ and $i$. The graph obtained in this case is $G_{10-11}$ shown in Figure 4.

The last option for $\left(\mathbf{d}_{a}, \mathbf{d}_{b}\right)$ is $(3,3)$. When $\left(\mathbf{d}_{a}, \mathbf{d}_{b}, \mathbf{d}_{c}, \mathbf{d}_{d}, \mathbf{d}_{e}\right)=(3,3,2,2,4)$, there are two possible degree sequences $(2,2,3,3,4)$ and $(2,3,3,3,3)$ for the partite $B$. Note that the degree sequence $(2,2,2,4,4)$ for the partite $B$ is covered in the case where there exist more than one vertex of degree 4 in one partite, where the resulting graph is $G_{10-8}$ in Figure 4.

In the case of $\left(\mathbf{d}_{f}, \mathbf{d}_{g}, \mathbf{d}_{h}, \mathbf{d}_{i}, \mathbf{d}_{j}\right)=(2,2,3,3,4)$, the vertex $j$ is adjacent to exactly one of $c$ or $d$, say $d$. In addition, $j$ is adjacent to $a$ and $b$. The vertex $h$ is adjacent to at most one of $c$ and $d$, thus its other neighbor is in $\{a, b\}$, say $a$. Similarly, $i$ has exactly one neighbor in $\{c, d\}$; thus, $i$ is also adjacent to $b$. Notice that if $i$ is adjacent to $d$, then $c$ is adjacent to $g$ and $h$ and $\{a, i, c\}$ is a minimal dominating set of size 3, contradiction. Therefore, $i d \notin E(G)$ and $i$ is adjacent to $c$. Note that if $d$ is adjacent to $g$, then $\{a, i, d\}$ is a minimal dominating set of size 3 , contradiction. Thus, $d$ is adjacent to $h$ and $g$ is adjacent to $c$. However, $\{b, h, c\}$ is a minimal dominating set of size 3 , contradiction. This case does not lead to any almost well-dominated graph.

We then suppose that $\left(\mathbf{d}_{f}, \mathbf{d}_{g}, \mathbf{d}_{h}, \mathbf{d}_{i}, \mathbf{d}_{j}\right)=(2,3,3,3,3)$. In this case, $d$ is adjacent to any two vertices in $\{g, h, i, j\}$, say $i$ and $j$. Then $c$ is adjacent to $g$ and $h$. The vertex $j$ is adjacent to one of $a$ or $b$, say $b$. Moreover, $a$ has at least one neighbor in $\{g, h\}$, say $g$. Now observe that $a$ is not adjacent to $i$, since otherwise $\{a, c, j\}$ is a minimal dominating set of size 3 , contradiction. Hence, $a$ is adjacent to $h$ and $b$ is adjacent to $i$. The graph obtained here is shown as $G_{10-9}$ in Figure 4.

Our main result in this section for almost well-dominated bipartite graphs with $\delta(G) \geq 2$ is stated in the following theorem.

Theorem 3.11. Let $G$ be a bipartite graph with $\delta(G) \geq 2$. Then $G$ is almost well-dominated if and only if $G$ is isomorphic to one of the following:

- If $|V(G)|=5$, then $G \cong G_{5}$.

- If $|V(G)|=6$, then $G \in\left\{G_{6-1}, G_{6-2}, G_{6-3}, G_{6-4}\right\}$.

- If $|V(G)|=7$, then $G \cong G_{7}$.

- If $|V(G)|=8$, then $G \in\left\{G_{8-1}, G_{8-2}, \ldots, G_{8-9}\right\}$.

- If $|V(G)|=10$, then $G \in\left\{G_{10-1}, G_{10-2}, \ldots, G_{10-16}\right\}$.

Proof. Let $G$ be a bipartite graph with $\delta(G) \geq 2$. We proceed the proof in two cases: $\delta(G) \geq 3$ and $\delta(G)=2$. The first case follows from Lemma 3.1 where $G \cong G_{6-1}$. For the second case, first note that the smallest order of an almost well-dominated bipartite graph with $\delta(G)=2$ is 5 and further it is shown by Corollary 2.4 that $|V(G)| \leq 10$. Then the result follows for each possible value of $|V(G)|$, separately, from Lemmas 3.2 to 3.4 and 3.7 to 3.10 .

For the converse direction $(\Leftarrow)$, the property of being almost well-dominated can be easily verified for the 31 graphs in Theorem 3.11 .

Acknowledgements. The support of the Scientific and Technological Research Council of Turkey (TUBITAK) under grant no. 118E799 is greatly acknowledged. The work of Didem Gözüpek was also supported by the BAGEP Award of the Science Academy of Turkey. 


\section{REFERENCES}

[1] J.E. Dunbar, L. Markus and D. Rall, Graphs with two sizes of minimal dominating sets. Congr. Numer. 111 (1995) $115-128$.

[2] B. Finbow, A. Hartnell and R. Nowakowsk, Well-dominated graphs: a collection of well-covered ones. Ars Comb. 25A (1988) 5-10.

[3] T.J. Gionet, E.L.C. King and Y. Sha, A revision and extension of results on 4-regular, 4-connected, claw-free graphs. Disc. Appl. Math. 159 (2011) 1225-1230.

[4] T. W. Haynes, S. Hedetniemi and P. Slater, Fundamentals of Domination in Graphs. In Vol. 208 of Monographs and Textbooks in Pure and Applied Mathematics, Marcel Dekker, New York, NY (1998).

[5] E.L.C. King, Characterizing a subclass of well-covered graphs. Congr. Numer. 160 (2003) 7-31.

[6] V.E. Levit and D. Tankus, Well-dominated graphs without cycles of lengths 4 and 5. Disc. Math. 340 (2017) $1793-1801$.

[7] W. McCuaig and B. Shepherd, Domination in graphs with minimum degree two. J. Graph Theory 13 (1989) 749-762.

[8] B. Reed, Paths, stars and the number three. Comb. Probab. Comput. 5 (1996) 277-295.

[9] J. Topp and L. Volkmann, Well covered and well dominated block graphs and unicyclic graphs. Math. Pannonica 1 (1990) 55-66.

[10] I.E. Zverovich and V.E. Zverovich, Locally well-dominated and locally independent well-dominated graphs. Graphs Comb. 19 (2003) 279-288. 